\title{
Tuberculosis of Wrist Presenting as Scaphoid Fracture: A Case Report
}

\author{
Diwakar Mishra, DNB, Ajay Gupta, MS \\ Department of Orthopaedics, Maulana Azad Medical College, New Delhi, India
}

\begin{abstract}
The scaphoid is an uncommon site for tuberculous infection. Clinical features and radiographic findings are not necessarily helpful and may lead to a delay in diagnosis and therefore poor treatment results. We report here a case of scaphoid tuberculosis, which presented as a scaphoid fracture, but then progressed to wrist arthritis. The patient was treated conservatively and had good functional outcome. To the best of our knowledge, such presentation has not been documented.
\end{abstract}

\section{INTRODUCTION}

The wrist is an uncommon site for tubercular infection ${ }^{1}$. Tubercular infection typically has a prolonged course and is rarely diagnosed before the development of full blown arthritis ${ }^{2,3}$. Patients usually presents with pain, swelling and restricted movement. Clinical features are not diagnostic and confusion is further compounded if there exists a history of trauma ${ }^{4}$.

\section{CASE REPORT}

A 22-year-old male, presented with complaints of gradual onset of pain and swelling in the right wrist for the previous

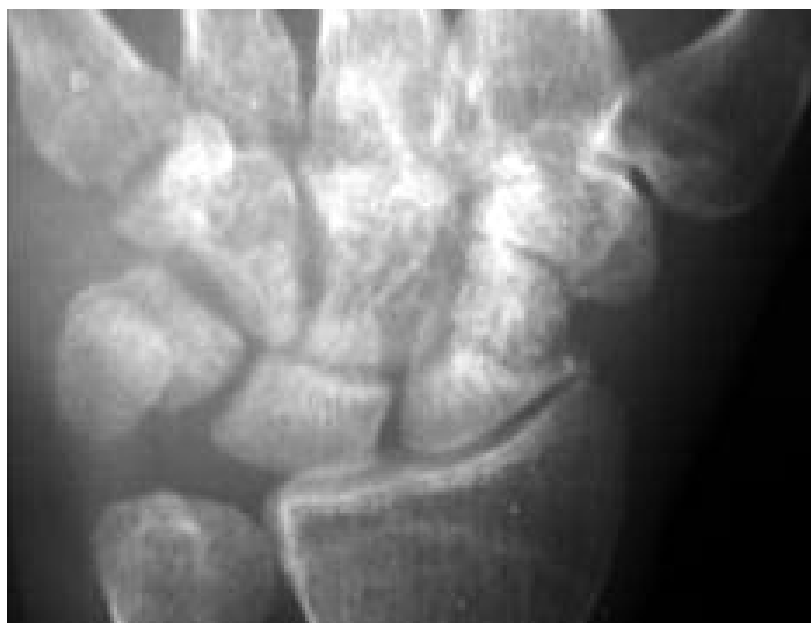

Fig. 1: Plain radiograph of the wrist showing scaphoid fracture. six months, with no history of trauma. The patient initially consulted a primary care physician where radiographs (Figure 1) of the wrist were obtained and a diagnosis of scaphoid fracture was made. The pain and swelling did not subside and instead continued to increase. At presentation to our clinic, the patient had gross swelling of the wrist and there was no history of tuberculous contact. Movement was painful and restricted in all planes, and radiographic examination revealed discontinuity in the scaphoid and decreased joint space (Figure 2). Laboratory results were as follows: haemoglobin-10.7 gm\%, Total Leucocyte Count-P (pro-myelocytes) 60\%, L (lymphocytes) 33\%, E (eosinophils) $7 \%$. Erythrocyte sedimentation rate (ESR) was $47 \mathrm{~mm}$ at the end of first hour. Synovial fluid examination revealed the presence of 3-4 pus cells/hpf (per high power field), and was negative for bacterial or fungal elements. Culture of the synovial fluid yielded no growth, but PCR (polymerase chain reaction analysis) of synovial fluid for mycobacterium tuberculosis was positive.

The diagnosis of wrist tuberculosis was made and the patient underwent Category I anti-tubercular treatment. A below elbow slab was applied for 6 weeks. Six months following the end of treatment, pain had subsided and the patient regained painless wrist motion although such motion was still somewhat restricted. Radiographs at treatment

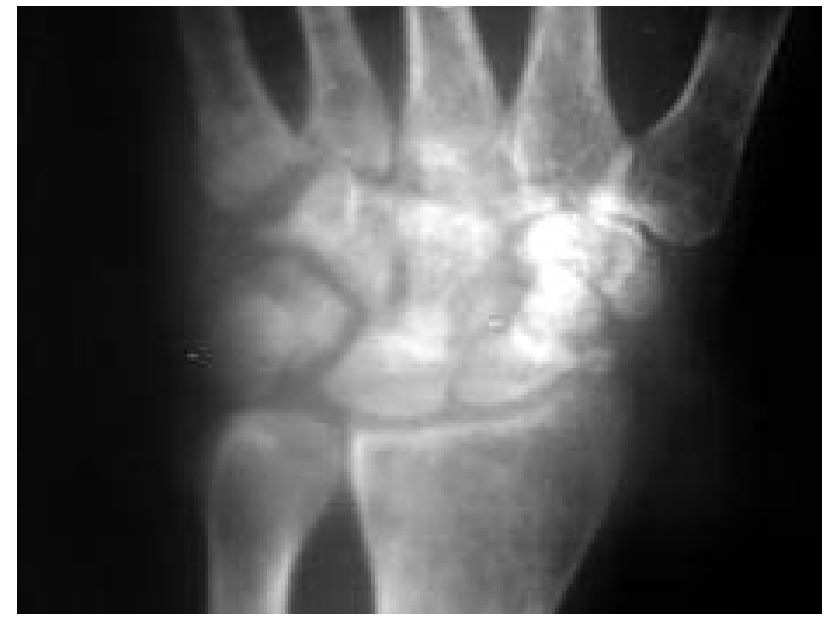

Fig. 2: Radiograph of wrist showing scaphoid nonunian and reduced joint space.

Corresponding Author: Diwakar Mishra, Department of Orthopaedics, Maulana Azad Medical College, Bahadur Shah Zafar Marg, New Delhi-110 002, India Email: diwakarlmishra@gmail.com 


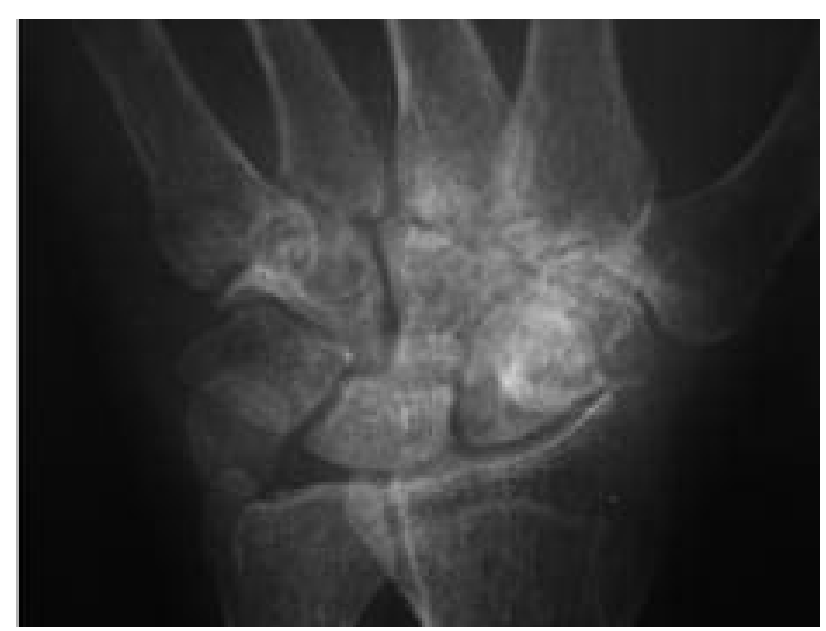

Fig. 3: Radiograph of the wrist taken 6 months after completion of tuberculosis treatment.

completion showed healed arthritis with resorption of proximal scaphoid (Figure 3).

\section{DISCUSSION}

Skeletal involvement occurs in $2-3 \%$ of patients suffering from tuberculosis ${ }^{5}$, with the wrist joint accounting for less than 1 per cent of those with skeletal tuberculosis ${ }^{1}$. Further, most cases have no history of pulmonary tuberculosis infection. Clinical symptoms are not definitive, leading to a delay in the establishment of conclusive diagnosis. Confusion is further compounded by the often misleading history of some traumatic event ${ }^{4}$. Phemister's triad, the presence of juxtaarticular osteoporosis, peripherally located osseous erosions, and gradual narrowing of the joint space, is suggestive of tubercular arthritis. Such characteristic findings are not present in every case. Early cases are more likely to be missed ${ }^{4}$. The radial side of the hand (distal radius, scaphoid, trapezium, capitate, bases of second and third metacarpals) is most frequently affected ${ }^{5}$. Involvement of carpus leads to marginal punched out lesions due to synovial involvement, or there may be cystic lesions due to intraosseous granulomas. 


\section{REFERENCES}

1. Bush DC, Schneider LH. Tuberculosis of the hand and wrist. J Hand Surg Am 1984; 9A: 391-8.

2. Evanchick CC, Davis DE, Harrington TM. Tuberculosis of Peripheral Joints: An Often Missed Diagnosis. J Rheumatol 1986; 13: $187-9$.

3. Tuli SM. Tuberculosis of The Skeletal System: 3rd Ed. Jaypee Brothers Medical Publishers (P) Ltd, New Delhi. 2004;153-8.

4. Yao DC, Sartoris DJ. Musculoskeletal tuberculosis. Radiol Clin North Am 1995; 33: 679-89.

5. Davidson P, Horowitz I. Skeletal tuberculosis. Am J Med 1970; 48: 77-84. 